\title{
International Session 1
}

\section{The role of IL-17 in chronic rhinosinusitis with nasal polyp}

\author{
Ji-Hun Mo ${ }^{1,2}$, Jun-Sang Bae ${ }^{2}$, Eun-Hee Kim², Ji Hye Kim ${ }^{2}$ \\ ${ }^{1}$ Department of Otorhinolaryngology, Dankook University College of Medicine, Cheonan, South Korea \\ ${ }^{2}$ Beckman Laser Institute Korea, Dankook University College of Medicine, Cheonan, South Korea
}

Objectives: Recently, IL-17 is known to play an important role in inflammatory disease including CRS. It is known to be increased in CRS with nasal polyp (NP). In this study, we evaluated the expression of IL-17 in CRS tissues and identified the cellular source of IL-17 in CRS with NP.

Methods: The expression of IL-17 in human CRS tissues were evaluated using PCR and immunohistochemistry $(\mathrm{n}=20$ each group). And the cellular sources of IL-17 were evaluated with double staining immunohistochemistry using confocal microscopy. IL-17A, CD68, CD163, ELA2 CD56, CD4 and CD11c antibodies were used to evaluate the cellular sources of IL-17. Eosinophilic and noneosinophilc polyps were evaluated ( $\mathrm{n}=5$ each sample).

Results: PCR and IHC showed that IL-17 expressions were increased in both eosinophilic and non- eosinophilic polyps, with significantly higher expression in noneosinophilic nasal polyp. Confocal staining revealed that about $60 \%$ of $\mathrm{CD} 68+$ M1 macrophage, $30 \%$ of ELA2+neutrophil and $10 \%$ of CD4 $\mathrm{T}$ cell coexpressed IL-17 irrespective of eosinophilic and noneosinophilc polyp, showing that M1 macrophage and neutrophil were major sources of IL17 in nasal polyp. NK cell, M2 macrophage and dendritic cells did not produce IL-17 in both eosinopholic and noneosinophilic nasal polyp. Conclusions: These data showed that iL-17 were highly expressed in nasal polyp and the major cellular sources are macrophage and neutrophil, suggesting that IL-17 might be used as target therapy in CRS with NP. In addition, M1 macrophage or neutrophil can also be used as a cellular target in CRSwNP. 